\title{
Ausgelagert und unsichtbar: Arbeitsbedingungen in der Reinigungsbranche
}

\author{
Karin Sardadvar ${ }^{\star}$
}

\section{Zusammenfassung}

Der Beitrag beschäftigt sich damit, in welcher Weise Reinigungsarbeit - als ein typischer Bereich sogenannter "Einfacharbeit" - in den vergangenen Jahrzehnten von Auslagerungen betroffen war und wie sie davon geprägt wurde. Er stellt aktuelle Branchencharakteristika und Beschäftigungsbedingungen zur Reinigungsbranche in Österreich im internationalen Kontext dar und argumentiert, dass die mit der Auslagerung verbundenen Veränderungen der Reinigungsarbeit problematische Arbeits- und Beschäftigungsbedingungen verstärken. So tragen insbesondere die verbreiteten Arbeitszeiten an den Tagesrändern zu einer Unsichtbarmachung der Arbeit bei, die einer mangelnden Anerkennung weiter Vorschub leistet. Die Tendenz zur gesellschaftlichen Unsichtbarmachung spiegelt sich daneben auch in der wissenschaftlichen und gesellschaftlichen (Nicht-)Zuwendung $z u$ "Einfachdienstleistungen" und der Reinigung im Speziellen wider. Der Beitrag geht weiters auf die Rolle des Wohlfahrtsstaates in der Gestaltung der Arbeits- und Lebensbedingungen von in "Einfachdienstleistungen" Beschäftigten ein. Abschließend weist er auf Ansatzpunkte für Verbesserungen auf unterschiedlichen Ebenen hin.

Schlagwörter: Reinigungsbranche, Dienstleistungssektor, Auslagerungen, Arbeitszeiten, Fragmentierung der Arbeit

\section{Outsourced and invisible: Working conditions in the cleaning sector}

\section{Abstract}

This contribution investigates how cleaning work, as a typical area of low-skill work, has been subject to outsourcing in recent decades and how this outsourcing has changed that work. It presents the current characteristics and employment conditions of the Austrian cleaning sector in the international context and argues that the changes to cleaning work linked to outsourcing, especially the prevalence of working times in the early morning and late afternoon or evening, contribute to making the work invisible and, in turn, little recognized. This invisibility is also reflected in the low societal and social-scientific attention paid to low-skill service work, and cleaning in particular. The article also discusses the role of the welfare state in designing the working and living conditions of workers in low-skill services. Finally, it highlights the possibilities for improvements on different levels.

Keywords: Cleaning sector, Service sector, Outsourcing, Working times, Fissuring of work

\footnotetext{
* Karin Sardadvar, Wirtschaftsuniversität Wien, Institut für Soziologie und Empirische Sozialforschung. E-Mail: karin.sardadvar@wu.ac.at

Ich bedanke mich bei Ingrid Mairhuber, Thomas Auinger, den ModeratorInnen und TeilnehmerInnen von Track \#9 des Momentum-Kongresses 2018 sowie den anonymen GutachterInnen für wichtige Hinweise und wertvolle Anmerkungen.
} 


\section{Einleitung: Die Unterhaltsreinigung als ausgelagerte „Einfachdienstleistung”}

In den vergangenen Jahrzehnten sind privatwirtschaftliche und öffentliche ArbeitgeberInnen in erheblichem Ausmaß dazu übergegangen, Arbeiten außerhalb ihrer Kerntätigkeiten auszulagern und an andere Unternehmen zu vergeben. Ein Bereich, in dem das sehr weitreichend geschehen ist - mit deutlichen Folgen für die Arbeits- und Beschäftigungsbedingungen -, ist die Unterhaltsreinigung.

Reinigungsarbeit ist ein Beispiel für das breitere Feld der sogenannten „Einfacharbeit“ - Tätigkeiten, für die keine konkrete Berufsausbildung erforderlich ist. Mit „Unterhaltsreinigung“ wird die regelmäßige Reinigung von Innenräumen, wie beispielsweise Büros, bezeichnet. Die Reinigung von Büroräumlichkeiten macht rund die Hälfte der Aktivitäten der gewerblichen Reinigungsbranche in Europa aus (EFCI 2017: 7). Bei der Unterhaltsreinigung handelt es sich um eine wichtige Subbranche des Wirtschaftszweiges Gebäudereinigung, der wiederum Teil der gewerblichen Reinigungsbranche als Ganzes ist. ${ }^{1}$

Der vorliegende Beitrag befasst sich mit der Unterhaltsreinigung als einem typischen Bereich weitgehend ausgelagerter „Einfachdienstleistungen“. Die in diesem Papier angesprochenen Befunde zur Reinigungsbranche basieren auf drei Quellen: internationaler wissenschaftlicher Literatur zur Branche, eigenen Forschungen mit KollegInnen zur Reinigungsbranche in Österreich und anderen EU-Ländern (dazu u. a. Holtgrewe/Sardadvar 2012) sowie meinem laufenden Forschungsprojekt SPLITWORK, ${ }^{2}$ das sich schwerpunktmäßig der Arbeitszeitform "geteilte Dienste“ in der Reinigungsbranche sowie in der mobilen Pflege und Betreuung in Österreich widmet.

Ziel des Beitrags ist es, eine in mehrfacher Hinsicht in die Unsichtbarkeit gerückte Arbeit ins Scheinwerferlicht zu stellen. Dabei sollen wesentliche Problematiken

1 In der Hierarchie und Bezeichnung der ÖNACEKlassifikation der Wirtschaftstätigkeiten in Österreich bildet sich die Reinigungsbranche wie folgt ab: ÖNACE Nr. 81 umfasst "Gebäudebetreuung; Garten- und Landschaftsbau“; diese teilt sich in Nr. 81.1 "Hausmeisterdienste" und Nr. 81.2 „Reinigung von Gebäuden, Straßen und Verkehrsmitteln“. Zur letzten Kategorie zählt die Allgemeine Gebäudereinigung, der auch die Unterhaltsreinigung angehört.

2 Split Shifts and the Fragmentation of Working Lives (SPLITWORK). Fördergeber: Austrian Science Fund (FWF): V-598. Laufzeit: 2018-2022. Für Projektinformationen siehe: https://bach.wu-wien.ac.at/d/research/projects/3187/. der Arbeits- und Beschäftigungsbedingungen vor dem Hintergrund von Auslagerungen und Wettbewerbsdruck, aber auch wohlfahrtsstaatlichen Entwicklungstendenzen, aufgezeigt und kritisch diskutiert werden. Darauf aufbauend werden Ansatzpunkte für Verbesserungen der Bedingungen benannt.

Im Folgenden skizziere ich zunächst das Feld der „Einfacharbeit“ (Abschnitt 2). Ich schildere dann, in welcher Weise die Reinigungsarbeit in den vergangenen Jahrzehnten von Auslagerungen betroffen war - und wie sie davon geprägt wurde (Abschnitt 3). Dazu stelle ich aktuelle Branchencharakteristika und Beschäftigungsbedingungen dar und diskutiere Aspekte der Prekarität der Beschäftigung (Abschnitt 4). Ich zeige danach, wie die mit der Auslagerung verbundenen Veränderungen der Reinigungsarbeit zur Unsichtbarmachung der Arbeit beitragen (Abschnitt 5). Die Unsichtbarmachung, so argumentiere ich, findet nicht nur am Arbeitsort statt, sondern spiegelt sich auch in der wissenschaftlichen und gesellschaftlichen (Nicht-) Zuwendung $\mathrm{zu}$ sogenannten „Einfachdienstleistungen“ und der Reinigung im Speziellen (Abschnitt 6), die auch Fragen sozialer Klassenverhältnisse aufwirft. Abschließend gehe ich auf die Rolle des Wohlfahrtsstaates in der Gestaltung der Arbeits- und Lebensbedingungen von Beschäftigten in der "Einfacharbeit“ ein und hebe Anknüpfungspunkte für Verbesserungen auf unterschiedlichen Ebenen hervor (Abschnitt 7).

\section{Schwierige Bedingungen für „einfache” Arbeit}

Als „Einfacharbeit“ bzw. im Dienstleistungsbereich auch „Einfachdienstleistungen“ werden Tätigkeiten mit geringen formalen Anforderungen im niedrig qualifizierten Bereich bezeichnet - etwa im Lager, in der Paketzustellung, an der Kasse oder eben in der Reinigung. Die Bedingungen in diesen „Einfachdienstleistungen“ werden als aufschlussreiches Phänomen der Tertiarisierung diskutiert (Esping-Andersen 1993; Bahl 2014a; Staab 2014a): Niedrige Entlohnung, atypische Beschäftigung und schlecht ausgebaute ArbeitnehmerInnenvertretung prägen die Arbeit dort, verbunden mit einer nur „losen Integration in die wohlfahrtsstaatlichen Absicherungssysteme" (Bahl/Staab 2010: 74). Gesellschaftliche Zuweisungen zu „Einfacharbeit“, insbesondere solcher mit prekären Beschäftigungsbedingungen, finden nach Geschlecht, Staatsbürgerschaft und Herkunft strukturell unterschiedlich statt (Baatz/ Schroth 2006: 286f.; Schroth/Schürmann 2006; Sardadvar 2016; Sardadvar et al. 2015). 


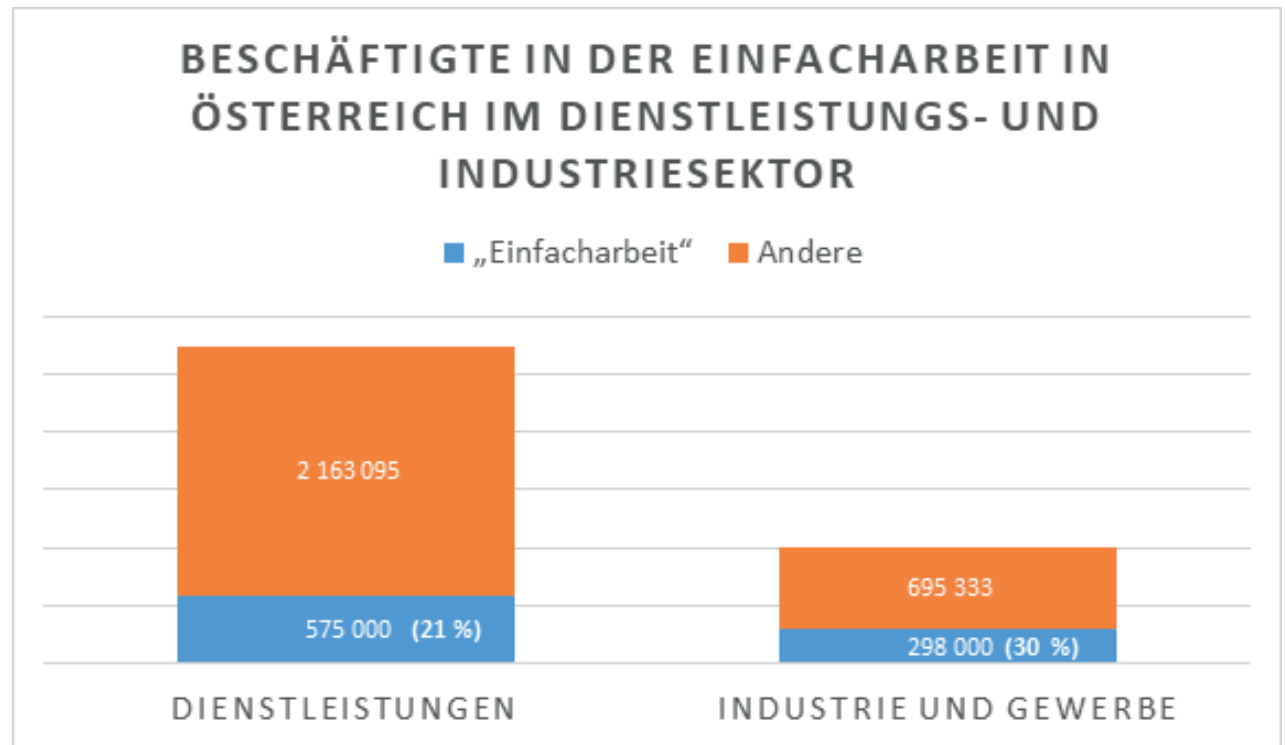

Quelle: Krenn (2015: 159), basierend auf Daten des Arbeitskräfteerhebungsdatensatzes (Mikrozensus), 2008-2012, Statistik Austria

Befunden für Österreich von Krenn (2015: 162) zufolge weist die Tätigkeit in Dienstleistungen auch innerhalb des Segments der „Einfacharbeit“ Nachteile gegenüber jener in Industrie und Gewerbe auf: Die Arbeitszeiten sind weniger stabil und weniger attraktiv, Vollzeitstellen sind seltener verfügbar, das Lohnniveau ist niedriger. Holtgrewe resümiert:

„Auf der einen Seite bieten die Einfachdienstleistungen Geringqualifizierten und anderen Menschen mit begrenzten Arbeitsmarktoptionen Zugangswege in reguläre Beschäftigung. Auf der anderen Seite stehen begrenzte Perspektiven, teilweise prekäre und nicht existenzsichernde Jobs mit eingeschränkten Entwicklungsmöglichkeiten" (Holtgrewe 2015: 10).

Krenn (2015: 158) beziffert den Anteil der unselbstständig Erwerbstätigen in Österreich, die in der „Einfacharbeit“ - definiert als Arbeitsplätze, die keine berufliche Ausbildung erfordern - beschäftigt sind, mit einem Anteil von rund 22 Prozent der Beschäftigten insgesamt bzw. 25 Prozent der unselbstständig Beschäftigten. ${ }^{3}$ Dabei sind zwei Drittel der „Einfacharbeit“ im Dienstleistungssektor zu finden und nur ein Drittel in Industrie und Gewerbe (Krenn 2015: 159; Krenn et al. 2014: 32). In absoluten Zahlen bedeutet dies, dass fast doppelt so viele „EinfacharbeiterInnen“ im Dienstleistungsbereich wie im Bereich Industrie und Gewerbe beschäftigt sind (rund 575.000 bzw. 298.000; siehe

3 Basierend auf Daten der Arbeitskräfteerhebung.
Grafik). Nichtsdestoweniger ist der Anteil der „Einfacharbeit" an der Gesamtbeschäftigung in Industrie als Gewerbe immer noch höher als im Dienstleistungssektor (3o bzw. 21 Prozent).

Mit den Auslagerungen der vergangenen Jahrzehnte waren dabei Bewegungen von "Einfacharbeit“ weg aus der Industrie hin in den Dienstleistungssektor verbunden (Krenn 2015: 159). Unter den zehn Branchen mit der größten (absoluten) Zahl an Beschäftigten in der „Einfacharbeit“ in Österreich liegt die Gebäudereinigung auf Platz 3 nach dem Einzelhandel und der Gastronomie (Krenn et al. 2014: 35f.). Die Gebäudereinigung weist mit 74,5 Prozent auch den höchsten Anteil an „EinfacharbeiterInnen“ auf (nach der kleinen Branche „Private Haushalte mit Hauspersonal“ mit insgesamt nur knapp 200 Beschäftigten) (Krenn et al. 2014: 36f.). ${ }^{4}$

Die Begriffe „Einfacharbeit“ und „Einfachdienstleistungen" sind inzwischen etabliert, aber meines Erachtens nicht glücklich gewählt, da sie diese wenig anerkannte Arbeit sprachlich weiter abwerten und die bezeichneten Tätigkeiten zudem keineswegs immer „einfach“ sind, und schon gar nicht „leicht“ im körperlichen Sinn. Kompetenzen, die sehr wohl benötigt

4 Zugrunde gelegt wurde der Anteil der Beschäftigten in Hilfs- und Anlerntätigkeiten, Durchschnitt 2008 bis 2012, Daten des Arbeitskräfteerhebungsdatensatzes (Mikrozensus) (Krenn et al. 2014). 
werden, werden jedoch nicht immer gesehen oder honoriert (Krenn 2015: 156). In der Reinigung zählen dazu zum Beispiel - nach Analysen der Schilderungen von BüroreinigerInnen selbst - soziale Fähigkeiten oder Gedächtnisleistungen, etwa in Bezug auf individuelle Präferenzen von Beschäftigten der Kundenunternehmen, deren Büros gereinigt werden (Sardadvar 2012). Andere Fähigkeiten, die ReinigerInnen ebenfalls selbst nennen, sind Flexibilität, Effizienz, Serviceorientierung und Kooperationsbereitschaft (Hohnen 2012: 26). Ganz allgemein stellen Leistungsbereitschaft und Belastbarkeit zentrale Anforderungen dar, die innerhalb der „Einfacharbeit" gestellt werden, und weitere Arbeitsintensivierung - also eine Zunahme der in einer bestimmten Arbeitszeit geleisteten Arbeit bzw. ein erhöhtes Arbeitstempo - prägt das Feld (Krenn 2015: 163).

Ich verwende die Begriffe „Einfacharbeit“ bzw. „Einfachdienstleistungen“ vor diesem Hintergrund unter Anführungszeichen (zum Begriff: Sardadvar/ Holtgrewe 2017: 216; Bahl 2014a: 11; Staab 2014a: 32f.). Dabei ist zu beachten, dass sich der Begriff der „Einfacharbeit" auf die Tätigkeit und nicht auf die Beschäftigten bezieht, die durchaus höhere Qualifikationen haben können, als die Tätigkeit verlangt (Krenn 2015: $152,160)$.

\section{Reinigungsarbeit: Ein typischer Fall für Auslagerung}

Die Reinigungsbranche zählte in den vergangenen Jahrzehnten zu den am schnellsten wachsenden Wirtschaftszweigen in Europa und Nordamerika (Aguiar 2006: 24; EFCI 2017; Holtgrewe et al. 2015). In Europa hat sich der jährliche Umsatz in der Branche nach Angaben des europäischen Dachverbands der Reinigungsbranche EFCI (European Federation of Cleaning Industries) von 1989 bis $2014 \mathrm{mehr}$ als verfünffacht: von 12,8 auf 73,9 Milliarden Euro pro Jahr (EFCI 2017: 4). ${ }^{5}$ Die Finanz- und Wirtschaftskrise ab dem Jahr 2007 hat die Branche vergleichsweise gut überstanden (ebd.). 1,7 Prozent der europäischen Beschäftigten arbeiten in der gewerblichen Reinigung, in Österreich 1,9 Prozent (Eurofound 2014: 1). ${ }^{6}$

Das Beschäftigungswachstum in der Branche kam jedoch nicht nur durch die Entstehung neuer Arbeits-

5 Datenbasis: 20 europäische Staaten (einschließlich Österreich)

6 Daten aus 2010. plätze zustande, sondern bildet zu einem wesentlichen Teil auch eine Verschiebung zwischen Branchen ab (Mayer-Ahuja 2003; Holtgrewe et al. 2015). Ab den 1970er-Jahren gingen Unternehmen in Industrieländern mehr und mehr dazu über, Tätigkeiten, die sie für ihr Kerngeschäft als peripher ansahen, auszulagern. Die Büroreinigung ist dafür ein typisches Beispiel. Auch öffentliche ArbeitgeberInnen begannen, Reinigungstätigkeiten vermehrt an private Unternehmen zu vergeben. Hinzu kamen Privatisierungen von zuvor öffentlichen Unternehmen, die ihrerseits die Beschäftigungsbedingungen der Belegschaften veränderten.

Vergaben nach außen versprachen Kostenersparnis, denn privatwirtschaftliche Reinigungsunternehmen kalkulierten mit niedrigeren Personalkosten - und somit Löhnen - als öffentliche ArbeitgeberInnen oder auch privatwirtschaftliche Unternehmen (Gather et al. 2005: 20). ${ }^{7}$ Ein weiteres Motiv für die Auslagerung von Tätigkeiten wie Reinigung, Hausbetreuung, Sicherheitsdienst, aber auch Buchhaltung, Telefondienst oder IT-Services war die Entledigung von Verantwortlichkeiten, die für Unternehmen damit verbunden sind, wenn sie ArbeitnehmerInnen selbst beschäftigen (Weil 2014: 3f.) - also beispielsweise, sich um Vertragsangelegenheiten, Lohnhöhen, Stundenabrechnungen und Abgaben kümmern zu müssen.

Die Zahl der Beschäftigten im österreichischen Reinigungsgewerbe beträgt, je nach Berechnungsmethode bzw. einbezogenen Branchen, zwischen rund 52.000 und 65.000 Personen (EFCI 2017: 14; AMS Österreich 2016; Ruhland et al. 2016). ${ }^{8}$ Der Anteil an

$7 \quad$ Angesprochen ist hier eine allgemeine, strukturelle Tendenz. Die Löhne in der ausgelagerten Reinigungsbranche sind nicht in jedem einzelnen Fall niedriger als bei Beschäftigung in-house, sondern dies ist vom jeweiligen Kollektivvertrag abhängig. So liegen die Einstiegsgehälter in Österreich für Reinigungskräfte bzw. RaumpflegerInnen laut Kollektivverträgen und Stellenausschreibungen beispielsweise im Handel bei 1.571 Euro brutto (Marchhart 2019) und damit in ähnlicher Höhe wie in der Reinigungsbranche selbst; beim Arbeitsmarktservice Österreich liegen sie bei 1.627,2 Euro brutto (AMS 2019) und in einer Tiroler Gemeinde bei 1.841,60 Euro brutto (Gemeinde Langkampfen 2019).

8 EFCI gibt eine Zahl von 52.00o Personen an. Diese entspricht in dieser Berechnung 1,53 Prozent der Beschäftigten und stimmt nicht ganz mit den oben erwähnten 1,9 Prozent der Beschäftigten von Eurofound überein, da unterschiedliche Arten von Daten und verschiedene Zeitpunkte zugrunde liegen (Eurofound 2014; EFCI 2017). Die Arbeitsmarktdatenbank des AMS weist rund 65.00o Beschäftigte im Wirtschaftszweig "Gebäudebetreuung, Garten- und Landschaftsbau“ aus (AMS Österreich 2016). 
Frauen (71 Prozent), an Personen mit nicht-österreichischer Staatsbürgerschaft (62 Prozent) sowie die Teilzeitquote (61 Prozent) in der Reinigungsbranche sind überdurchschnittlich hoch (Ruhland et al. 2016: 4).

Die Marktdurchdringung von Reinigungsunternehmen wird in Europa auf etwa 65 Prozent geschätzt, d.h. die privatwirtschaftliche Reinigungsindustrie geht davon aus, 65 Prozent des Marktes für Reinigungsdienstleistungen bereits $\mathrm{zu}$ bedienen. Dabei liegen hohe nationale Unterschiede vor: Die Marktdurchdringung reicht von 43 Prozent in der Tschechischen Republik bis zu 85 Prozent in den Niederlanden. In Österreich wird dabei im Vergleich mit 82 Prozent die zweithöchste Marktdurchdringung angegeben (EFCI 2017: 6f.). ${ }^{9}$ Wenn dieser Wert auch Schwierigkeiten bei der Berechnung unterliegt, weist er doch darauf hin, dass die Auslagerung der Reinigung in Österreich weit fortgeschritten ist und nur mehr rund ein Fünftel der Reinigungstätigkeiten in-house, also in privatwirtschaftlichen Unternehmen oder öffentlichen Organisationen selbst, verrichtet wird. Auf europäischer Ebene ist die Marktdurchdringung im ausgelagerten Bereich von 52 Prozent im Jahr 1994 über 6o Prozent im Jahr 2005 auf 65 Prozent im Jahr 2014 angestiegen (EFCI 2017: 6f.). Für Österreich fehlen in dieser Darstellung die Angaben für 1994 (2005: 80 Prozent; 2014: 82 Prozent); nach ExpertInneneinschätzung ging die Auslagerungsentwicklung in der Reinigung in Österreich aber eher früh und eher schnell vonstatten (Holtgrewe/ Sardadvar 2011; Kirov 2011).

Infolge der weitreichenden Marktdurchdringung ist die Branche in Europa heute von starkem Wettbewerb geprägt. Der Wettbewerb, verbunden mit einem hohen Personalkostenanteil, führt zu dem Versuch, bei den Personalkosten einzusparen, etwa über Billigangebote und Arbeitsverdichtung, und zu betrügerischen Praktiken bei einigen inkorrekten Reinigungsanbietern (Holtgrewe/Sardadvar 2011, 2012; Scandella 2010b; Adam 2017).

David Weil (2014) identifiziert in seinem Buch The Fissured Workplace Auslagerungen und Vergaben als Spielarten eines fissuring der Beschäftigung - also eines Zerklüftens, Spaltens, Rissigwerdens, einer Fragmentierung. ${ }^{10}$ Gebäudereinigung und Sicherheitsdienste bilde-

9 Datenbasis: 19 europäische Staaten (einschließlich Österreich); Jahr: 2014.

10 Franchising, Offshoring und länger werdende Wertschöpfungsketten mit immer mehr SubauftragnehmerInnen sind weitere Varianten des fissuring (Weil 2014). ten dabei im Prozess der Fragmentierung die Vorhut, und gerade bei ReinigerInnen und HausbetreuerInnen lässt sich anhand der internationalen Literatur rekonstruieren, wie das Auslagern dieser Arbeit in schlechtere Vertragsbedingungen und niedrigere Löhne mündete (Weil 2014: 9of.; Dryden/Stanford 2012; Aguiar/Herod 2006a).

Insgesamt waren die Folgen von Auslagerungen, Privatisierungen und Deregulierung für die Reinigungsbranche und ihre Beschäftigungsbedingungen international beträchtlich (Aguiar/Herod 2006a, b). Die Reinigung ist in diesem Kontext besonders interessant, weil sie, wie deutsche Forscherinnen festhalten, schon früh eine "bedenkliche Vorreiterolle“ (Gather et al. 2005: 209; dazu auch Baatz/Schroth 2006: 282) hinsichtlich Flexibilisierung und Prekarisierung von Beschäftigung einnahm. Aguiar und Herod (2006b: 4f.) führen in internationaler Zusammenschau die folgenden hauptsächlichen Transformationen von Reinigungsarbeit in den vergangenen Jahren an, die sie als Folge neoliberaler politischer Tendenzen wie Deregulierung und Privatisierung betrachten: Die Arbeit wurde intensiviert (d. h. es ist mehr Arbeit in weniger Zeit zu verrichten), die Löhne sanken und es wurde gleichzeitig versucht, die Arbeit in der Branche zunehmend zu professionalisieren (z. B. in Hinblick auf Ausbildungen, Verberuflichung, Zertifizierungen).

Staab (2014b: 3) identifiziert für „Einfachdienstleistungen“ wie die Reinigung drei charakteristische Tendenzen der Rationalisierung: Standardisierung, Verdichtung und Universalisierung. Das bedeutet, die Beschäftigten haben weniger Freiraum in ihrer Arbeit (Standardisierung), sie müssen immer schneller arbeiten (Verdichtung) und sie werden zunehmend universal für unterschiedlichste Tätigkeiten eingesetzt (Universalisierung). Diese Entwicklungen stehen nun aber mit den oben genannten Professionalisierungsbestrebungen im Konflikt und begünstigen Dequalifizierung, analysiert Staab:

„Standardisierungsmaßnahmen führen formale Professionalisierungsversuche ad absurdum, weil Arbeit an Komplexität verliert. Sie setzen eine Dequalifizierungsdynamik in Gang, die sich in den realisierbaren Löhnen niederschlägt (...)" (Staab 2014b: 3).

Was aus einer betriebswirtschaftlichen Perspektive rational sein mag, ist in sozialer Hinsicht problematisch, streicht Weil denn auch in Bezug auf sein Konzept des fissured workplace hervor. Er führt drei Gründe für soziale Bedenken an: 
„There are three reasons we should worry about the social consequences of the fissured workplace. First, it often undermines compliance with basic labor standards. Second, chopping employment into pieces makes production coordination harder and results in a problem economists call externalities that can result in accidents, injuries, and fatalities. Third, there are distributional consequences of the fissured workplace, shifting surplus generated by businesses away from the workforce and to investors"(Weil 2014: 16).

Die Fragmentierung der Arbeit bedroht demnach die Einhaltung von Beschäftigungsstandards, erhöht die Verletzungsgefahr und trägt dazu bei, dass weniger von der Wertschöpfung bei den Beschäftigten ankommt. In der Tat verzeichnen Forschungen in der Reinigungsbranche zahlreiche illegale und unlautere Praktiken, auch in Österreich - etwa falsche Lohneinstufungen, inkorrekte Stundenabrechnungen oder Scheinselbstständigkeit (Adam 2017; Bretterbauer et al. 2010). Insgesamt sind die Beschäftigungsbedingungen in der heutigen Reinigungsbranche international problematisch und vielfach prekär (Aguiar/ Herod 2006a, b; Holtgrewe/Sardadvar 2012). Darauf gehe ich im folgenden Abschnitt näher ein.

\section{Reguliert und doch prekär: Beschäftigungsbedingungen in der Reinigungsbranche}

Im europäischen Vergleich von 33 Wirtschaftszweigen zeigt die Reinigungsbranche in mehrfacher Hinsicht besonders ungünstige Beschäftigungsbedingungen (Eurofound 2014): Dazu zählen ein hoher Anteil an befristeten Verträgen und Teilzeitverträgen (darunter auch unfreiwillig niedrige Stundenausmaße), niedrige Löhne, hohe Gesundheits- und Sicherheitsrisiken sowie dürftige Entwicklungs- und Aufstiegsperspektiven.

Insgesamt legt der Forschungsstand nahe, dass die Beschäftigung in der Reinigungsbranche eine der niedrigsten Positionen in der Beschäftigungshierarchie darstellt (Abbasian/Hellgren 2012: 161; Bradley 2006: 76). Mit nationalen und kontextuellen Unterschieden weisen die Beschäftigungsbedingungen hohe Prekarität auf. Diese wird begleitet von sozialer Ungleichheit auch innerhalb der Branche - etwa nach Geschlecht und Herkunft (z. B. Sardadvar 2016; Schürmann 2013; Schroth/Schürmann 2006).

Viele dieser Probleme sind auch für Österreich festzustellen. Und doch ist die Reinigungsbranche in Österreich keine durchwegs schlechte Arbeitgeberin.
Sie ist sozialpartnerschaftlich weitreichend geordnet und gestaltet; die Löhne und Beschäftigungsbedingungen sind wesentlich durch Kollektivverträge geregelt (Holtgrewe/Sardadvar 2011). Hält man sich vor Augen, dass parallel zur gewerblichen Reinigungsbranche auch noch ein großer informeller, unregulierter Reinigungssektor existiert - der vom Staat als „Privatsache“ der Privathaushalte geduldet wird (ebd.) -, bietet die gewerbliche Reinigung potenziell stabile und leicht verfügbare Arbeitsplätze mit niedriger, aber erwartbarer Entlohnung. Denn festzuhalten ist an dieser Stelle, dass der Zustand der Arbeit in den „Einfachdienstleistungen“ politisch durchaus gestaltbar ist, was enorme Unterschiede in den Beschäftigungs- und Arbeitsbedingungen innerhalb der EU widerspiegeln (Holtgrewe 2015: 12; Holtgrewe/ Sardadvar 2012).

Doch auch in der österreichischen Reinigungsbranche finden sich innerhalb des regulierten Rahmens Aspekte von Prekarität. Zu ihnen zählen die schon erwähnten inkorrekten und illegalen Praktiken von Unternehmen bei Arbeitsverträgen, Einstufungen und Abrechnungen und vielem mehr (Adam 2017; Bretterbauer et al. 2010). Hinzu kommt, dass die gewünschten und tatsächlichen vertraglichen Arbeitszeitausmaße in Österreich in der Branche erheblich auseinanderklaffen (Michenthaler et al. 2013). Daneben besteht unter den Beschäftigten große Unzufriedenheit mit den weit verbreiteten Arbeitszeiten an den Tagesrändern (siehe dazu Abschnitt 5) (Bretterbauer et al. 2010).

Vor allem aber sind die Löhne niedrig. Sie sind zwar wie erwähnt durch die hohe Kollektivvertragsabdeckung zumindest berechenbar. Auch sind sie in der Höhe vergleichbar mit den Löhnen in den Lehrberufen am unteren Ende der Entlohnung, wie zum Beispiel jenen von FriseurInnen. Aber sie müssen jedenfalls zum Teil als Niedriglöhne betrachtet werden.

Betrachten wir das Lohnniveau etwas eingehender: Die kollektivvertraglichen Stundenlöhne in der Denkmal-, Fassaden- und Gebäudereinigung betragen 2019 je nach Lohngruppe von 8,98 bis 10,96 Euro brutto. UnterhaltsreinigerInnnen, zu denen BüroreinigerInnen gehören, sind dabei in der Lohngruppe 6 - der untersten Lohngruppe - angesiedelt.

Die Niedriglohngrenze liegt nach gängiger internationaler Definition bei zwei Dritteln des Medianlohns. Sie betrug laut nationaler Berechnungsmethode im Jahr 2014 9,24 Euro brutto (ohne Mehr- und Überstunden) pro Stunde für Österreich (Geisberger 2017). Damit 
liegen aktuell die niedrigsten beiden kollektivvertraglichen Stundenlöhne im Jahr 2019 (also vier Jahre nach der letzten Berechnung der Niedriglohngrenze) für UnterhaltsreinigerInnen mit 8,98 Euro unter der Niedriglohngrenze von 9,24 Euro."

Nach Daten des Arbeitsklimaindex zählt die Reinigung außerdem zu jenen Branchen in Österreich, in denen ein besonders hoher Anteil der Beschäftigten mit dem Einkommen nicht oder kaum auskommt. 16 Prozent der Reinigungskräfte geben an, dass ihr Einkommen nicht ausreicht, weitere 59 Prozent, dass es gerade ausreicht. Personen, die von ihrem Einkommen nicht leben können, weisen im Arbeitsklimaindex wiederum insgesamt niedrige Werte bei der Lebenszufriedenheit und bei der Zufriedenheit mit ihrer sozialen Position auf. 84 Prozent von ihnen gehen außerdem nicht davon aus, später von ihrer Pension leben zu können (AK OÖ 2018: 2).

Die niedrigen Löhne haben in der Tat weitreichende Folgen für die soziale Absicherung. Nicht nur für den laufenden Lebensunterhalt ist der Lohn knapp, sondern auch für den künftigen: Wie Bahl (2014b) für Niedriglöhne in Deutschland analysiert, trifft auch für Österreich zu, dass sich das niedrige Lohnniveau in der Pensionsleistung niederschlägt und dazu führen kann, dass Beschäftigte im Alter armutsgefährdet sind oder in Armut leben. Dies gilt auch im Fall von Arbeitslosigkeit, ist doch das Arbeitslosengeld bei 55 Prozent des Nettolohns angesetzt.

Bei all diesen Aspekten - dem aktuellen Einkommen und Transfereinkommen wie Arbeitslosengeld und Pensionsleistung - ist zudem stets zu bedenken, dass Beschäftigte in der Reinigung oft teilzeitbeschäftigt sind und daher lediglich Teilzeitlöhne erhalten. Denn insbesondere in der Unterhaltsreinigung und speziell bei weiblichen Beschäftigten ist Teilzeitbeschäftigung verbreitet - vielfach unfreiwillig: 65 Prozent der Beschäftigten in der Reinigung waren im Jahr 2014 in Österreich teilzeitbeschäftigt (dies entspricht etwa dem EU-Schnitt) (EFCI 2017: 19f.). Gleichzeitig wünschen sich in den EU-28 Beschäftigte in der Reinigung deutlich öfter als andere Beschäftigte ein höheres als ihr aktuelles Arbeitsstundenausmaß (Eurofound 2014: 3).

11 Kollektivvertragliche Stundenlöhne nach Lohngruppen ab 1.1.2019: Lohngruppe 1: 10,96 Euro; Lohngruppe 2: 9,95 Euro; Lohngruppe 3: 9,71 Euro; Lohngruppe 4: 9,42 Euro; Lohngruppe 5: 9,08 Euro; Lohngruppe 6: 8,98 Euro (vida/WKO 2019).
Die Reinigungsbranche weist international in beträchtlichem Ausmaß prekäre Beschäftigungsbedingungen auf (Holtgrewe/Sardadvar 2012; Eurofound 2014). Inwieweit die Beschäftigung im österreichischen Reinigungsgewerbe prekär ist, ist dennoch nicht pauschal für die gesamte Branche zu beantworten. Es hängt u. a. mit der jeweiligen kollektivvertraglichen Einstufung und damit der konkreten Lohnhöhe, der Korrektheit des konkreten Unternehmens und dem Beschäftigungsverhältnis (z. B. gewünschte oder aber unfreiwillig niedrige Stundenanzahl) zusammen. Generell, so zeigt eine aktuelle Studie von Allmendinger et al. (2018) für Deutschland, sind Menschen, die in der „Einfacharbeit" beschäftigt sind, in unterschiedlichen Konstellationen von prekärer Beschäftigung und prekären Haushaltslagen besonders stark vertreten.

In der sozialwissenschaftlichen Literatur bestehen unterschiedliche Sichtweisen auf das große Theorieund Forschungsfeld der Prekarität wie auch auf die tatsächliche empirische Bedeutung des Konzeptes in der Gegenwart - auf diese Debatte wird hier nicht näher eingegangen. Nach dem Zugang von Klaus Dörre ist Prekarität kein absolutes Kriterium, sondern richtet sich nach den gesellschaftlichen Orientierungsmustern und bestehenden Rahmenbedingungen.

„Nach einer Jenaer Arbeitsdefinition [der Autor bezieht sich auf sein Forschungsumfeld an der FriedrichSchiller-Universität Jena; Anm. K.S.] gilt ein Erwerbsverhältnis dann als prekär, wenn es nicht dauerhaft oberhalb eines von der Gesellschaft definierten kulturellen Minimums existenzsichernd ist und deshalb bei der Entfaltung in der Arbeitstätigkeit, gesellschaftlicher Wertschätzung und Anerkennung, der Integration in soziale Netzwerke, den politischen Partizipationschancen und der Möglichkeit zu längerfristiger Lebensplanung dauerhaft diskriminiert" (Dörre 2014: 4).

Auf die große Gruppe der in den niedrigsten Lohnstufen eingestuften ReinigerInnen in Österreich - Schätzungen der Sozialpartner zufolge sind etwa 60 bis 80 Prozent der Beschäftigten allein der niedrigsten Lohngruppe zugeteilt (Holtgrewe/Sardadvar 2011: 18; unveröffentlichte Recherchedaten) - trifft die hier angesprochene unzureichende Existenzsicherung mit den angeführten Folgen tendenziell zu. Sehr typisch gerade für die Reinigung ist außerdem der von Dörre angeführte Mangel an gesellschaftlicher Wertschätzung und Anerkennung. Er wird durch eine Dynamik der Unsichtbarmachung der ReinigerInnen und ihrer Arbeit verschärft, die ich am Beispiel der Büroreinigung im Folgenden beschreibe. 


\section{Ausgelagert und aufgesplittert: Die Unsichtbarmachung der Reinigung}

„Ich bin im Rahmen langjähriger Forschungsarbeiten noch keiner Form von Lohnarbeit begegnet, die so hartnäckig ignoriert, so konsequent unter dem Konsens des Verschweigens begraben wurde, wie das Putzen" (Duda 1990: 1).

Das Herauslösen der ReinigerInnen aus den Stammbelegschaften von privaten Unternehmen oder öffentlichen ArbeitgeberInnen forcierte eine Unsichtbarmachung der ReinigerInnen und ihrer Arbeit (dazu auch Bahl 2014a: 112ff.). Wie das obige Zitat von Silke Duda aus einer Pionierstudie zu Reinigerinnen 1990 zeigt, hat eine gesellschaftliche Ignoranz der Reinigung Tradition. Die mit den Auslagerungen verbundenen Veränderungen haben ihr weiter Vorschub geleistet.

Die Reinigung von Büros und Arbeitsstätten findet heute vielfach in den frühen Morgenstunden oder $a b$ dem späten Nachmittag statt - vor Dienstbeginn und nach Dienstschluss anderer Beschäftigter, wenn die Stammbelegschaften ihre Büros noch nicht betreten oder bereits verlassen haben (EFCI 2017: 17; Michenthaler et al. 2013: 14ff.). So treffen Belegschaften von Unternehmen mit ReinigerInnen beauftragter Reinigungsdienstleister selten zusammen: Die Arbeitszeiten wurden in den vergangenen Jahrzehnten vielerorts so gestaltet, dass die Anwesenheiten der Belegschaft des KundInnenunternehmens und jener des Reinigungsbetriebes sich möglichst wenig überschneiden. Auch die Arbeitszeiten in der Reinigung im öffentlichen Sektor gleichen sich dabei tendenziell jenen in der Privatwirtschaft an (Gather et al. 2005: 150).

(Vermutete) Störungen der Stammbelegschaft durch Tätigkeiten wie Staubsaugen und Bodenwischen sollen vermieden werden. Hier fallen Bestrebungen, nach denen sowohl die Büroangestellten als auch die BüroreinigerInnen jeweils effizient arbeiten sollen, zusammen. Den oder die ReinigerIn mit Namen zu kennen oder kurz das Büro zu verlassen, damit er oder sie seine oder ihre Arbeit verrichten kann, ist an vielen Arbeitsstätten unüblich. Die Folge dieser Bedingungen ist eine Unsichtbarmachung der extern beschäftigten Reinigungskraft und ihrer Tätigkeit. Gesehen wird allenfalls das Ergebnis ihrer Arbeit - das saubere Büro, die aufgeräumte Küche, das geputzte WC - bzw. das, was als nicht zufriedenstellend erledigt negativ auffällt (Duda 1990: 1; Karafyllis 2013). Aber ReinigerIn und Unternehmensangestellte/r gelangen wenig in direkten Kontakt, und sie sind, da bei unterschiedlichen ArbeitgeberInnen beschäftigt, in der Regel auch keine
KollegInnen mehr. Für die ReinigerInnen sind damit, wie angesprochen, häufig Teilzeitbeschäftigungen mit entsprechend niedrigen Einkommen, aber auch soziale Isolation bei der Arbeit und niedrige Anerkennung verbunden (Holtgrewe/Sardadvar 2011, 2012).

Dass dies nicht immer so war, ist wenig bekannt oder wird wenig bedacht. Mit der Übernahme der Reinigung durch spezialisierte privatwirtschaftliche Reinigungsunternehmen und eine damit verbundene neue Form der KundInnenbeziehung wurden die Arbeitszeiten von BüroreinigerInnen verstärkt an die Tagesränder geschoben - an den frühen Morgen sowie den späten Nachmittag und Abend. Damit zu diesen Zeiten gereinigt werden kann, hat etwa jede/r zehnte/r Beschäftigte/r in der Reinigungsbranche - und ein wesentlich höherer Anteil in der Subbranche Unterhaltsreinigung - sogenannte geteilte Dienste: ${ }^{12}$ Das bedeutet, der Arbeitstag ist durch eine meist mehrstündige unbezahlte Pause geteilt, die Beschäftigten reisen zwei Mal an einem Tag zu ihrem Arbeitsort (dem gleichen oder verschiedenen) an, haben vier Anfahrtswege. Oder sie verbringen, wenn der Weg nach Hause zu weit ist, die unbezahlte Pause beispielsweise im öffentlichen Raum oder in einer Gaststätte.

Die Arbeit an den Tagesrändern (frühmorgens, abends) ist interessanterweise in Österreich besonders verbreitet: Hier finden nur 8 Prozent der Reinigungsarbeit während der üblichen Bürozeiten statt. 20 Prozent werden am frühen Morgen verrichtet, 70 Prozent in den Abendstunden (EFCI 2017: 17). ${ }^{13}$ Das ist jedoch nicht unvermeidlich: Reinigung war in der Vergangenheit stärker in die gewöhnlichen Tagesarbeitszeiten integ-

12 Quelle: Projekt SPLITWORK; unveröffentlichte Berechnungen auf Basis von Daten von Statistik Austria.

13 Diese Daten unterliegen allerdings, insbesondere im internationalen Vergleich, einigen Unklarheiten in Bezug auf, erstens: die Details der Erhebung, und zweitens: die in den Staaten jeweils angelegte Definition von „Tagesarbeitszeit“. Die Definitionen im Bericht werden wie folgt und lediglich vage angegeben (EFCI 2017:17): mises"

- daytime cleaning: „during the occupation of the pre-

- early in the morning: „Immediately after the night period and before the beginning of ,usual ' working hours (generally from $5 / 6$ to $8 / 9$ am)“

- in the late afternoon / beginning of the evening: „After the end of the , usual' working hours and before the beginning of the night period (generally from 5/6 to 8/9 pm)“

Die Aussagekraft der Daten sollte also nicht überschätzt werden; sie können aber eine ungefähre Orientierung in Bezug auf die Verbreitung von Tagesarbeitszeit in verschiedenen Staaten geben. 
riert, und auch heute erreichen nordische Staaten wie Schweden und Finnland (wieder) Tagesarbeitsanteile von über 70 Prozent (ebd.; im Detail zu Norwegen Torvatn/Lamvik 2011; Sardadvar 2017). Die Arbeitszeiten in der Büroreinigung sind, so schließe ich daraus, nicht zuletzt eine Frage der kulturellen Gepflogenheiten und eingebürgerten Erwartungen - und damit auch eine Sache der Gestaltung und des Veränderungswillens.

Eine große Rolle für die gegenwärtige Arbeitszeitstruktur in der Reinigung spielt indes die durch die Auslagerungen verbreitete Konstellation des Dienstleistungsdreiecks. Wie auch in anderen Dienstleistungsbranchen ist die Arbeit in der gewerblichen Reinigung im Dienstleistungsdreieck zwischen Beschäftigten, KundInnen und ArbeitgeberInnen angesiedelt (u. a. Leidner 1996; Korczynski 2002) (oder auch, angesichts länger werdender Wertschöpfungsketten, im Dienstleistungsviereck; Kirov 2015).

Das prägt nicht nur die Organisation der Arbeit und die Arbeitsbedingungen entscheidend, sondern auch das Vorgehen und die Position der Sozialpartnerschaft: So ist beispielsweise bei den verbreiteten geteilten Diensten und den Arbeitszeiten an den Tagesrändern festzustellen, dass ArbeitgeberInnen- und ArbeitnehmerInnenvertretungen ihre Interessen bis zu einem gewissen Grad teilen: Beide Seiten betrachten es als wünschenswert, von den fragmentierten Arbeitszeiten wegzukommen - Gewerkschaften zum Wohl von ArbeitnehmerInnen, Wirtschaftstreibende zur Senkung des Organisationsaufwands sowie um angesichts von Rekrutierungsschwierigkeiten zur Attraktivität der Beschäftigung beizutragen (Holtgrewe/Sardadvar 2011, 2012). Nach einer aktuellen Erhebung unter 51 Reinigungsunternehmen in Österreich wünschen sich mehr als 6o Prozent dieser Betriebe Tagesarbeitszeit (ÖSB 2018: o. S.). Unter den Beschäftigten von Reinigungsunternehmen hätten laut einer Befragung von 52 ReinigerInnen in Österreich 69 Prozent gern Tagesarbeitszeit zwischen 8 und 18 Uhr (Michenthaler et al. 2013: 14ff.). Doch dazwischen steht als Barriere der KundInnenwunsch nach möglichst unsichtbarer Arbeit, auf den beide Seiten - ArbeitgeberInnen- und ArbeitnehmerInnenvertretungen - verweisen. Mit anderen Worten: Gestaltungswille und auch Konsens wären durchaus vorhanden, doch beide Seiten der Interessenvertretung sehen sich vom Wunsch der KundInnen blockiert. Beispiele wie jene aus den nordischen Staaten legen jedoch nahe, dass andere Einstellungen zur Reinigung, auch seitens der KundInnen, möglich sind.
Karafyllis spricht in einer philosophischen Betrachtung des Putzens noch einen ganz anderen Aspekt an - den, dass die Reinlichkeit der BüronutzerInnen tendenziell sinkt, wenn kein Kontakt zur Reinigungskraft besteht:

„Das Verhalten ist aber auch nicht fair gegenüber den Putzkräften, die immer weniger im persönlichen Kontakt mit dem Büronutzer für einzelne Büros zuständig sind, sondern von den Facility Management-Firmen ein sogenanntes, Revier'zugeteilt bekommen, in dem sie sauber zu machen haben. Dies geschieht sehr früh am Morgen oder sehr spät am Abend, damit man die, wirklich'Arbeitenden nicht bei ihrer Arbeit stört. Durch den Mangel an persönlichem Kontakt sinkt auch die Schamgrenze des Büro- und Kaffeeküchenverschmutzers, weil er niemanden mehr hat, vor dem er sich schämen muss" (Karafyllis 2013: 130).

Es geht hier also auch um kulturelle und interaktive Aspekte der Gestaltung der Arbeit: Wie verhält sich die Stammbelegschaft, wenn sie Kontakt zur Reinigungskraft hat oder eben nicht? Wie beeinträchtigend ist es tatsächlich, wenn während der Büroarbeit kurz das Zimmer abgestaubt wird? Wie irritierend wäre es, fünf Minuten das Büro zu verlassen, damit ein/e Reiniger/in seine/ihre Arbeit verrichten kann, wenn es der tägliche, gewohnte Normalfall wäre?

Die Unsichtbarkeit der Reinigung ist nicht nur in Hinblick auf die individuelle Isolation und mangelnde Anerkennung der Beschäftigten folgenreich. Sie erschwert vielmehr auch die betriebliche und gesellschaftliche Anerkennung dieser Arbeit. Mangelnde Anerkennung wiederum trägt neben "harten Fakten“ wie den Lohnniveaus in den Kollektivverträgen für die Gebäudereinigung auf einer symbolischen Ebene dazu bei, die Löhne niedrig zu halten. Gleichzeitig ist die Unsichtbarkeit der Arbeit eng mit den Arbeitszeiten an den Tagesrändern verknüpft, die Teilzeitbeschäftigungsverhältnisse und damit nicht-existenzsichernde Entlohnungen begünstigen.

\section{6. Übersehene Arbeit eines \\ Dienstleistungsproletariats}

Die Reinigungsarbeit bleibt nicht nur an den konkreten Orten unsichtbar, an denen sie verrichtet wird. Auch Gewerkschaften haben der Reinigung lange Zeit vergleichsweise wenig Beachtung geschenkt (Herod/ Aguiar 2006: 173) (siehe dazu Abschnitt 7). Die Sozialwissenschaften haben die Reinigungsarbeit und andere „Einfachdienstleistungen“ ebenfalls mit wenig Aufmerksamkeit bedacht. Bahl (2014a: 19) konstatiert, dass die 
„einfachen Dienstleistungen“ lange „[i]im toten Winkel industriesoziologischer Analyse verblieben sind“.

Dafür sind mehrere Gründe auszumachen. Wenngleich in den vergangenen Jahren das Forschungsinteresse für Reinigungsarbeit und andere „Einfachdienstleistungen“ merklich angestiegen ist (exemplarisch: Holtgrewe/Sardadvar 2012; Schürmann 2013; Bahl 2014a; Staab 2014a), fällt die Aufmerksamkeit für diese wichtige und große Branche doch auffallend gering aus. Das mag zum einen mit gesellschaftlich unangenehmen Konnotationen der Reinigung mit Schmutz zu tun haben (Karafyllis 2013). Zum anderen hängt es zweifelsohne mit der allgemeinen Geringschätzung von Tätigkeiten, die vielfach unbezahlt von Frauen erledigt wurden und werden, wie Care-Arbeit oder eben Haus- und Reinigungsarbeit, zusammen (Baatz/Schroth 2006: 296; England 2005).

Aber auch ein klassenspezifisches Bias von WissenschafterInnen in der Zuwendung zu „Einfachdienstleistungen" und Reinigungsarbeit ist möglicherweise am Werk. SoziologInnen sind selbst Gesellschaftsmitglieder, sie haben durch ihre eigene Klassenzugehörigkeit blinde Flecken und produzieren klassenspezifische Forschungsinteressen bzw. -ignoranzen (dazu auch Altreiter 2017: 40; 2018: 263). So schreibt Bourdieu mit Kollegen:

„Unter allen kulturellen Vorgaben, die möglicherweise in die Interpretation des Soziologen eingehen, ist der Einfluß des Klassenethos, des organisierenden Prinzips für die Aneignung der anderen unbewußten Modelle, am systematischsten und zugleich am besten verborgen" (Bourdieu et al. 1992: 84).

Die Reinigung ist nun eine vornehmlich von niedrig qualifizierten oder aus anderen Berufen kommenden, mehrheitlich weiblichen, zu hohen Anteilen nach Österreich eingewanderten Personen verrichtete Tätigkeit (Ruhland et al. 2016; Holtgrewe/Sardadvar 2011, 2012; Sardadvar 2016). Sie ist vielfach unsichtbar, wird in anderen Kontexten oft unbezahlt erledigt, wird gesellschaftlich wenig wertgeschätzt und ist zudem weder neue noch ungewöhnliche Arbeit. Es liegt nahe, dass all dies dazu beiträgt, dass diese Arbeit unverhältnismäßig wenig Beachtung erfährt.

Die Soziologie tat sich aber auch aus konzeptionellen Gründen lange schwer mit der Betrachtung von "Einfachdienstleistungen“ (Staab 2014a; Bahl 2014a). Die Arbeits- und Industriesoziologie hat sich bis in die 1970er-Jahre stark mit dem Klassenbegriff beschäftigt, bis in den 1980er-Jahren die Perspektive zur Individualisierung und dem Milieubegriff hin wechselte.
Damit wanderte der Blick von strukturellen sozialen Ungleichheiten zu eher individuellen Lebensstilen und -entscheidungen. Für die Betrachtung eines entstehenden "Service-Proletariats“ (Esping-Andersen 1993) bot dies keine günstige Voraussetzung.

Einer Beschäftigung mit „Einfacharbeit“ im Allgemeinen abträglich waren auch Beiträge zur Wissensgesellschaft und zum technologischen Wandel, die davon ausgingen, dass Einfacharbeit immer unwichtiger werden würde, da sie zum einen von wissensintensiver Arbeit und zum anderen von technischem Fortschritt ersetzt werden würde. Gegen die Annahme einer solchen Marginalisierung von "Einfacharbeit" führt Krenn (2015) ins Treffen, dass die Beschäftigung im Bereich „einfacher" Dienstleistungsarbeit in den vergangenen Jahren gewachsen ist (dazu auch Holtgrewe et al. 2015) und dass nach wie vor ein erheblicher Anteil von Arbeitsplätzen, für die keine Berufsausbildung erforderlich ist, besteht und weiterhin bestehen bleiben wird.

Doch auch die Einordnung der „Einfachdienstleistungen" in den Klassenbegriff ist schwierig: Die niedrig entlohnte, oft weibliche Kraft in der „einfachen" Dienstleistungsarbeit entspricht nicht unbedingt einem verbreiteten Verständnis von Arbeiterklasse mit der Konnotation des typisiert männlichen Industriearbeiters (Staab 2014a: 35). Die „Einfachdienstleistungen“ sind im Begriff der Arbeiterklasse nicht automatisch enthalten, sie werden aber auch nicht im Begriff der Dienstleistungsarbeit, der zunächst stark von der Wissens- und Angestelltenarbeit dominiert war, erfasst (Bahl 2014a: 9).

Deutsche WissenschafterInnen haben in den vergangenen Jahren für die Auseinandersetzung mit den Beschäftigten in "Einfachdienstleistungen“ vor diesem Hintergrund verstärkt den Begriff des „Dienstleistungsproletariats" eingebracht (Bahl 2014a; Staab 2014a) - aufbauend auf dem frühen Hinweis des Wohlfahrtsstaatsforschers Gøsta Esping-Andersen (1993) auf die Entstehung eines "Service-Proletariats“ im Rahmen post-industrieller Klassenstrukturen.

Staab argumentiert, dass vieles, was der traditionellen Arbeiterklasse mit Fokus auf Industriearbeit zugeschrieben wurde, auf die „einfache Dienstleistungsarbeit" durchaus zutrifft, wenn man ein Klassenverständnis bemüht, das den Begriff "Proletariat“ weiter fasst als „exklusiv als Bezeichnung für eine spezifische Klassenlage mit historischer Mission und gemeinsamem (revolutionärem) Bewusstsein“ (Staab 2014a: 380). Er nennt vor dem Hintergrund 
seiner empirischen Forschung einige Merkmale, die die beiden Gruppen vereinen: die Körperlichkeit der Arbeit, eine Resignation in Bezug auf ihre Situation sowie der niedrige Lohn und eine Lebensführung ohne Rücklagen (Staab 2014a: 372ff.).

Die Frage nach der Rolle Angestellter für den Klassenbegriff wurde schon früher verschiedentlich aufgenommen: In den 196oer-Jahren hatten WissenschafterInnen, darunter Eduard März (1968) in Österreich, die These von den Angestellten als neuer Arbeiterklasse bearbeitet und die Unterschiede zwischen Angestellten und ArbeiterInnen untersucht (Hofmann/Weiss 2018: 239). Ob Angestellte oder Gruppen unter ihnen als Proletariat anzusehen seien, wird nach Hans Paul Bahrdt schon diskutiert, seit Angestellte in nennenswerter Anzahl in der Gesellschaft vertreten sind. Er selbst schreibt im Jahr 1962:

„Die Angestelltenmassen, die weiter wachsen, stellen nach wie vor ein gesellschaftliches Problem dar. Es ist nicht $z u$ bestreiten, daß sich manche Merkmale der proletarischen Lage heute häufiger bei ihnen finden als vor wenigen Jahrzehnten. (...) Mehr denn je müssen sie ihre Arbeit als fremdbestimmt, als Ausschnitt aus einer im ganzen unverständlich bleibenden Welt verstehen, die dennoch alle ihre vorhandenen Kräfte rücksichtslos dienstbar macht" (Bahrdt 1962: 24).

Dennoch bleiben die Beschäftigten in „Einfachdienstleistungen“ erstens äußerst heterogen - nicht zuletzt hinsichtlich ihrer Bildungsabschlüsse -, und sie sind zweitens auch formal nicht notwendigerweise als ArbeiterInnen tätig. ${ }^{14}$ Diese Aspekte und die Frage, ob sich Beschäftigte in „Einfachdienstleistungen“ selbst in der Zuordnung zum „Dienstleistungsproletariat“ wiederfinden und auf dieser Grundlage etwa auch solidarisieren würden, gilt es aus meiner Sicht in Bezug auf das Konzept des Dienstleistungsproletariats noch verstärkt zu diskutieren.

\section{Veränderungspotenziale und Verantwortungen}

Geteilte Dienste und Arbeitszeiten an den Tagesrändern in der Reinigung werden in Österreich heute von VertreterInnen von Unternehmen, Gewerkschaften und Wirtschaftskammer vielfach als selbstverständlich

14 Reinigungskräfte in Österreich sind arbeitsrechtlich bis zur Position des Vorarbeiters oder der Vorarbeiterin als ArbeiterInnen eingestuft. Mit dem Aufstieg in die nächste Hierarchiestufe, die erste Leitungsebene des Objektmanagements bzw. der Objektleitung, erfolgt auch der „Aufstieg“ in den Angestelltenstatus. und bedauerlicherweise unvermeidbar angesehen. Vergleiche mit anderen Staaten und Rückblicke in die Vergangenheit lassen aber, wie ich argumentiert habe, annehmen, dass hier durchaus erheblicher Gestaltungsspielraum besteht. Gesellschaftliche Bewusstseinsarbeit und die Adressierung des Problems in der Kommunikation mit KundInnenunternehmen (auch der öffentlichen Hand) sind hier ein möglicher Ansatzpunkt. Vorteile von Tagesarbeitszeit für die KundInnen werden in Kampagnen und Erhebungen immer wieder gesammelt, sie werden aber den KundInnen noch nicht ausreichend vermittelt (ArbeitGestalten 2017a: 21; Ramioul 2012: 9f.; ÖSB 2018: o. S.). ArbeitGestalten (2017a: 21) listet auch Schritte auf, die den Übergang zur Tagesreinigung in Abstimmung mit den KundInnen erleichtern können.

Da Unternehmen jedenfalls nicht am KundInnenwunsch vorbeiagieren wollen, stellt sich weiterführend die Frage, welche Möglichkeiten es für den Staat bzw. auf der kollektivvertraglichen Ebene gibt, hier gestaltend einzugreifen - beispielsweise mit Veränderungen bei den vorgeschriebenen Nachtzuschlägen zwischen derzeit 21 und 6 Uhr (die sich auf die Arbeitszeiten auswirken würden) oder mit gesetzlichen oder kollektivvertraglichen Einschränkungen bei geteilten Diensten.

Gewerkschaften sind generell gefordert, Beschäftigte in "Einfachdienstleistungen“ stärker wahrzunehmen, zu erreichen, zu organisieren und entsprechend zu vertreten. Die Organisierung einer sowohl örtlich als auch zeitlich verstreut tätigen Belegschaft mit fragmentierten Beschäftigungsverhältnissen, hoher Fluktuation und unterschiedlichen Sprachkenntnissen ist schwierig, aber nicht mehr als Ausnahmefall anzusehen. Wie ich andernorts in Bezug auf die Haltung der Gewerkschaften in der Reinigungsbranche ausgeführt habe (Sardadvar 2016), gilt es für Gewerkschaften auch, Gefahren der Reproduktion gesellschaftlicher Ungleichheit in ihrer eigenen Arbeit (etwa Rassismen, Geschlechterstereotype) stärker zu reflektieren und zu vermeiden.

Savage (2006) weist vor dem Hintergrund erfolgreicher großer Kampagnen wie der Bewegung „Justice for Janitors“, die in den 1980er-Jahren in den USA und Kanada entstand, darauf hin, was ihrer Analyse zufolge erfolgreiche ArbeitnehmerInnenvertretung im Bereich der Gebäudereinigung und -betreuung ausmacht. Ausschlaggebend ist demnach eine gelungene Kombination von lokaler Spezifizität einerseits und ausreichender Allgemeinheit andererseits zur Erreichung einer kritischen Masse: 
„The key, therefore, seems to be the ability to develop campaigns and structures which provide sufficient flexibility to incorporate local specifity, yet which also provide enough collective mass and centralized coordination as to allow workers to stand up to their employers" (Savage 2006: 23of.).

Schroth (2008: 203) analysiert als wichtiges Charakteristikum der Tätigkeit der US-amerikanischen Dienstleistungsgewerkschaft SEIU, die die Kampagne "Justice for Janitors“ vorangetrieben hat, „das widersprüchliche Nebeneinander einer zentralistisch und hierarchisch strukturierten Organisation (...) und einem basisdemokratisch angelegten Konzept von Mitgliederpartizipation“. Sie hält die ArbeitnehmerInnen in prekären „Einfachdienstleistungsjobs“ insgesamt durchaus für organisierbar und warnt in Bezug auf prekäre Arbeit vielmehr davor, „im Lamento einer Elendsberichterstattung zu verharren“ (ebd.: 206). In diesem Zusammenhang wäre allerdings auch notwendig, dass Gewerkschaften zunehmend von der verbreiteten Orientierung am männlich dominierten Normalarbeitsverhältnis abweichen (ebd.: 206f.).

Angelehnt an das Beispiel der Bewegung "Justice for Janitors“ wurde 2004 in Großbritannien die Kampagne „Justice for Cleaners" durchgeführt. Dieser „transnationale Austausch“ gewerkschaftlicher Aktionen stimmt auch Scandella (2010a: 208; Übersetzung K.S.) optimistisch in Hinblick auf die Zukunft der ArbeitnehmerInneninteressenvertretung selbst in den schwierigen Dienstleistungsbranchen.

Weil (2014) sieht Veränderungspotenzial und -notwendigkeit in Hinblick auf sein Konzept des problematischen fragmentierten fissured workplace auf gesetzlicher Ebene: Große Unternehmen müssten verpflichtet werden, Verantwortung auch für die ArbeitnehmerInnen der von ihnen beauftragten Unternehmen zu übernehmen:

\section{„(...) innovative solutions could be created by re-esta-} blishing that lead companies have some shared responsibility for the conditions arising in the network of workplaces they influence through their other activities" (Weil 2014: 287).

Unternehmen sollten sich demzufolge an den Kosten der sozialen Folgen ihrer Strategien beteiligen müssen. Sie sollten die Arbeitsbedingungen an den von ihnen mitgeschaffenen Arbeitsplätzen ebenso im Auge behalten müssen, wie sie auch durchaus in der Lage sind, die erbrachte Leistungsqualität $\mathrm{zu}$ überprüfen. Beispiele für konkrete mögliche Maßnahmen, die verantwortungsvolle mächtige Organisationen in Hinblick auf Subunternehmen und ähnliche Konstruktionen übernehmen (müssen) könnten, sind nach Weil (2014): die Errichtung von internen Trainingsstrukturen für affiliierte Organisationen, die Aufnahme detaillierter Beschäftigungsstandards in Leistungsverträge, der Aufbau formaler Monitoring-Systeme, die die Einhaltung der Beschäftigungsstandards in den beauftragten Betrieben überprüfen, und eine strikte Abgrenzung von SubauftragnehmerInnen oder Franchisebetrieben, die in fragwürdige Aktivitäten verwickelt sind. In manchen großen Unternehmen werden solche und ähnliche Forderungen heute im Rahmen von Nachhaltigkeitsprogrammen auf freiwilliger Basis bereits verfolgt.

$\mathrm{Zu}$ fragen ist jedoch auch nach der Verantwortung des Wohlfahrtsstaates, die hier eine mehrfache ist: Zunächst hat der Wohlfahrtsstaat als Arbeitgeber, indem er Tätigkeiten wie die Reinigung nach außen vergeben hat, diese Beschäftigten aus seiner Obhut und Kontrolle entlassen. Wenn Weil oben fordert, dass große privatwirtschaftliche Unternehmen Verantwortung und Monitoring für die Beschäftigungsbedingungen in den von ihnen beauftragten Unternehmen übernehmen sollten, wenn also an die Fürsorgepflicht auch über das eigene Unternehmen hinaus appelliert wird, so gilt dies wohl für den Arbeitgeber Wohlfahrtsstaat erst recht. Der Wohlfahrtsstaat ist bzw. seine öffentlichen Einrichtungen sind auch zu KundInnen geworden, wo sie z. B. Reinigungsdienstleistungen von anderen Unternehmen einkaufen. Hier besteht Verantwortung, aber auch Potenzial für Gestaltung und Verbesserung, indem öffentliche AuftraggeberInnen veränderte Arbeitszeiten, Beschäftigungsbedingungen etc. einfordern könnten.

Einen wichtigen Schritt hat in diesem Zusammenhang die österreichische Gewerkschaft vida im Vorjahr in Bezug auf Vergabekriterien für die öffentliche Hand gesetzt: Um das Bestbieterprinzip zu stärken (in Abgrenzung vom Billigstbieterprinzip), wurde für die Branchen Reinigung und Bewachung ein Katalog für faire Vergabekriterien erstellt (ÖGB 2018). Darin werden für die Reinigung beispielsweise die folgenden Qualitätskriterien angeführt, die bei der Vergabe berücksichtigt werden sollen: „Einsatz qualifizierter ReinigerInnen“ (Schulungen, Nachschulungen), "Qualitätsmanagement“ (regelmäßige Evaluierung von Arbeitsprozessen), „Geringe Personalfluktuation“ oder „Reservekapazitäten“ (Personal) (ÖGB 2018: 8ff.). Die für den Katalog erarbeiteten Kriterien sind nicht bindend; sie sollen bei Ausschreibungen Orientierung 
dahingehend bieten, wie sich soziale Aspekte bei der Vergabe einfangen lassen. Auch auf europäischer Ebene wurde ein Leitfaden zur Vergabe abseits des Billigstbieterprinzips im öffentlichen und privatwirtschaftlichen Sektor erstellt (Uni Europa/EFCI 2016), für den ArbeitgeberInnen- und ArbeitnehmerInnenvertretung zusammengearbeitet haben. Literatur zur Vergabepraxis in der Gebäudereinigung in Deutschland weist in der Tat mehrfach darauf hin, dass SachbearbeiterInnen und EntscheidungsträgerInnen in der Vergabe Bedarf nach Informationen und Fortbildung in Bezug auf soziale Kriterien haben (ArbeitGestalten 2017b: 16; Eigenstetter et al. 2016: 65).

Der Wohlfahrtsstaat ist generell überall dort an den bestehenden Bedingungen beteiligt, wo er auch im regulierten Bereich, wie in der gewerblichen Reinigung, als Regulierungsebene oder als Kunde Aspekte der Prekarität zulässt oder mitgestaltet: etwa in den atypischen und unsozialen Arbeitszeiten, den Niedriglöhnen, den unfreiwilligen Teilzeitverträgen oder den illegalen Unternehmenspraktiken.

Hinzu kommt, wie Bahl (2014b) für Deutschland argumentiert, dass der aktivierende Wohlfahrtsstaat das Entstehen von niedrig entlohnten unqualifizierten Stellen forciert hat. Sie analysiert:

„Der gegenwärtige Wohlfahrtsstaat hat über sein arbeitsmarktpolitisches Prinzip der Aktivierung einen entscheidenden Anteil an der Herausbildung dieser, Jedermannsarbeitsmärkte. (...) Niedriglohneinkommen und geringe Alterssicherung sind erste Hinweise, dass sich im Bereich, einfacher' Dienste nicht nur eine Situation multipler Benachteiligung abzeichnet, sondern auch ihre Verfestigung" (Bahl 2014b: 2).

Bahl spricht damit zum einen an, dass die Politik des aktivierenden Wohlfahrtsstaates, etwa durch das Prinzip der Lohnaufstockung im Sozialleistungssystem, verbunden mit dem steigenden Zwang, jede Arbeit annehmen zu müssen, ArbeitnehmerInnen bzw. Arbeitsuchende in prekäre Stellen mit niedrigen Einkommen drängt. ${ }^{15}$

15 GeringverdienerInnen (Personen mit einem Einkommen bis zu zwei Dritteln des mittleren Einkommens) können in Deutschland Anspruch auf Sozialleistungen im Sinne von Lohnaufstockungen im Rahmen von Hartz IV haben. In Österreich war dies im Rahmen der bisherigen Mindestsicherung auch möglich. Durch die noch unbefristete Bezugsmöglichkeit von Notstandshilfe ist der Druck, sehr niedrig entlohnte Beschäftigungen anzunehmen, hier aber noch verhältnismäßig gering (Stand: Mai 2019).
Zum anderen bezieht sich Bahl darauf, dass der deutsche Mindestlohn, der diese Branchen erfasst, nicht dazu ausreichen wird, später eine Rente $\mathrm{zu}$ erzielen, von der man leben kann. ${ }^{16}$ „Sein Niveau lässt erwarten, dass viele Arbeitnehmer auch nach dem Ausscheiden aus dem Berufsleben auf wohlfahrtsstaatliche Grundsicherung angewiesen sein werden“ (Bahl 2014b: 2). Zu ergänzen ist, dass dies neben der Lohnhöhe auch mit der Ausgestaltung des deutschen Rentensystems zusammenhängt, das im internationalen Vergleich (auch im Vergleich mit Österreich) eine sehr niedrige Ersatzrate aufweist, d. h. dass ein besonders niedriger Prozentsatz des Erwerbseinkommens durch die Rente ersetzt wird.

Der Wohlfahrtsstaat prägt somit mit seiner aktivierenden Arbeitsmarktpolitik und spezifischen Ausgestaltung von Sozialleistungen auch die Situation von Personen in „Einfachdienstleistungen“. Gleichzeitig ist er gefordert, bestehende Härten bzw. Lücken in der Existenzsicherung langfristig abzufedern bzw. zu schließen. Dabei gelten die Bedingungen in Deutschland nicht in gleicher Weise in Österreich: Unterschiede bestehen im Mindestlohn, im Pensionssystem sowie in der Arbeitslosenversicherung und der bisherigen Mindestsicherung - aber die Tendenzen und Gefahren sind ähnliche. Das österreichische Pensionssystem etwa weist hier weniger Härten auf als das deutsche, doch auch für das österreichische Pensionssystem gilt, dass ein niedriges (Teilzeit-)Einkommen über Jahre zu einer sehr geringen bzw. nicht-existenzsichernden Pensionsleistung führt. In diesem Kontext sind nicht zuletzt die bis vor Kurzem von der bis Mai 2019 amtiert habenden österreichischen ÖVP-FPÖ-Regierung geplante Abschaffung der Notstandshilfe und die jüngst erfolgte Ersetzung der Mindestsicherung durch die Sozialhilfe höchst problematisch.

Die Bedingungen in und Aussichten für die „Einfachdienstleistungen“ sind gegenwärtig alles andere als einfach. Mein zentraler Punkt in diesem Beitrag ist jedoch, dass die Arbeits- und Beschäftigungsbedingungen in der Reinigungsbranche trotz allem nicht durch den Prozess der Auslagerung determiniert sind, sondern dass Prozesse der Unsichtbarmachung der Arbeit und die damit verbundenen Abwärtsspiralen

16 Der allgemeine Mindestlohn in Deutschland beträgt derzeit 8,84 Euro brutto pro Stunde. (Nochmals zum Vergleich: Der niedrigste kollektivvertragliche Lohn für UnterhaltsreinigerInnen in Österreich beträgt derzeit 8,98 Euro). 
hinsichtlich Arbeitszeiten, Anerkennung etc. gestaltbar und veränderbar sind. Dazu muss die Reinigungsarbeit nicht notwendigerweise erneut „insourced“, also wiedereingegliedert werden - ein unrealistisches Szenario, für das es aber im Übrigen vereinzelt durchaus auch erfolgreiche Beispiele gibt (Engartner 2016). Gestaltungspotenzial besteht auch unter den gegenwärtigen Bedingungen auf verschiedenen Ebenen - auf wohlfahrtsstaatlicher, gesetzlicher, sozialpartnerschaftlicher, kollektivvertraglicher, gewerkschaftlicher, betrieblicher, kultureller und interaktiver Ebene -, Ebenen, die miteinander in Austausch stehen und an denen parallel angesetzt werden kann.

\section{Literatur}

Abbasian, S./Hellgren, C. (2012): Working Conditions for Female and Immigrant Cleaners in Stockholm County An Intersectional Approach. Nordic Journal of Working Life Studies, 2 (3), 161-181.

Adam, G. (2017): Fraudulent Contracting of Work: Industrial Cleaning Sector. Dublin: Eurofound.

Aguiar, L.L.M. (2006): Janitors and Sweatshop Citizenship in Canada. In: Aguiar, L.L. M./Herod, A. (Hg.): The Dirty Work of Neoliberalism. Cleaners in the Global Economy. Malden, Oxford, Carlton: Blackwell Publishing, 16-36.

Aguiar, L.L.M./Herod, A. (Hg.) (2006a): The Dirty Work of Neoliberalism. Cleaners in the Global Economy. Malden, Oxford, Carlton: Blackwell Publishing.

Aguiar, L.L.M./Herod, A. (2006b): Introduction: Cleaners and the Dirty Work of Neoliberalism. In: Dies. (Hg.): The Dirty Work of Neoliberalism. Cleaners in the Global Economy. Malden, Oxford, Carlton: Blackwell Publishing, $1-10$.

AK OÖ (2018): Arbeitsklimaindex Newsletter 03/2018. Linz: Kammer für Arbeiter und Angestellte für Österreich.

Allmendinger, J./Jahn, K./Promberger, M./Schels, B./Stuth, S. (2018): Prekäre Beschäftigung und unsichere Haushaltslagen im Lebensverlauf: Gibt es in Deutschland ein verfestigtes Prekariat? WSI-Mitteilungen, 71 (4), 259-269.

Altreiter, C. (2018): Soziale Klasse in der Arbeitssoziologie. Zur Relevanz eines totgesagten Begriffs. Österreichische Zeitschrift für Soziologie, 43 (3), 251-266.

Altreiter, C. (2017): Subjekt und Klasse. Zur Dialektik von Position und Disposition junger IndustriearbeiterInnen. Dissertation, Universität Wien.

AMS (2019): Kollektivvertrag 2019 für die DienstnehmerInnen des Arbeitsmarktservice. Kollektivvertrag vom 4.12.2018. Online: https://www.ams.at/content/dam/download/allgemeine-informationen/oo1_kv_ams.pdf [07.05.2019].

AMS Österreich (Hg.) (2016): Gender und Arbeitsmarkt. Geschlechtsspezifische Informationen nach Berufsbereichen. Wien: Arbeitsmarktservice Österreich.
ArbeitGestalten (2017a): Branchenreport Gebäudereinigung. Arbeitszeiten und Arbeitsverhältnisse. Berlin: ArbeitGestalten.

ArbeitGestalten (2017b): Können öffentliche Ausschreibungen Einfluss auf die Beschäftigungsbedingungen in der Gebäudereinigung und Schulverpflegung nehmen? Berlin: ArbeitGestalten.

Baatz, D./Schroth, H. (2006): Du putzt Deutschland: Die Prekarisierung von Erwerbsarbeit in der Reinigungsbranche. In: Degener, U./Rosenzweig, B. (Hg.): Die Neuverhandlung sozialer Gerechtigkeit. Feministische Analysen und Perspektiven. Wiesbaden: Springer VS, 281-299.

Bahl, F./Staab. P. (2010): Das Dienstleistungsproletariat. Theorie auf kaltem Entzug. Mittelweg 36, 19 (6), 66-93.

Bahl, F. (2014a): Lebensmodelle in der Dienstleistungsgesellschaft. Hamburg: Hamburger Edition.

Bahl, F. (2014b): Am Rande der Servicewelt: Das Problem der Solidarisierung in den „einfachen“ Diensten. Gegenblende - das DGB-Debattenportal. Online: http:// gegenblende.dgb.de/++co++446289ea-7638-11e4-82of52540066f352 [16.07.2018].

Bahrdt, H.P. (1962): Die Angestellten. In: Feuersenger, M. (Hg.): Gibt es noch ein Proletariat? Dokumentation einer Sendereihe des Bayerischen Rundfunks. Frankfurt am Main: Europäische Verlagsanstalt, 15-24.

Bradley, H. (2016): Gender and Work. In: Edgell, S./Gottfried, H./Granter, E. (Hg.): The SAGE Handbook of the Sociology of Work and Employment. London: Sage, 73-92.

Bourdieu, P./Chamboredon, J.-C./Passeron, J.-C. (1991): Soziologie als Beruf: wissenschaftstheoretische Voraussetzungen soziologischer Erkenntnis. Berlin: de Gruyter.

Bretterbauer, B./Burgholzer, C./Höpfl, T. (2010): Wie hilfreich ist Diversity Management? Die Arbeitssituation von Frauen und Migrantinnen im Reinigungsgewerbe. WISO, 33 (4), 135-147.

Dörre, K. (2014): Prekarität als Konzept kritischer Gesellschaftsanalyse - Zwischenbilanz und Ausblick. Ethik und Gesellschaft, 2/2014. Online: http://www.ethik-undgesellschaft.de/ojs/index.php/eug/article/viewFile/22014-art-1/26 [16.07.2018].

Dryden, R./Stanford, J. (2012): The unintended consequences of outsourcing cleaning work. Ottawa: Canadian Centre for Policy Alternatives.

Duda, S. (1990): Die unsichtbaren Arbeiterinnen. Frauen in Reinigungsberufen. Bielefeld: Kleine Verlag.

EFCI (2017): The cleaning industry in Europe. An EFCI Survey. Edition 2016 (Data 2014). Brussels: European Federation of Cleaning Industries (EFCI).

Eigenstetter, M./Jähn, V./Langhoff, T./Luven, N./Wallmeier, M. (2009): Reinigungsunternehmen und öffentliche Auftraggeber in der Vergabepraxis. Reihe Study, Nr. 313. Düsseldorf: Hans-Böckler-Stiftung.

Engartner, T. (2009): Kehrt der Staat zurück? Rekommunalisierungen in den Aufgabenbereichen Entsorgung und Gebäudereinigung. Zeitschrift für öffentliche und gemeinwirtschaftliche Unternehmen, 32 (4), 339-355. 
England, P. (2005): Emerging Theories of Care Work. Annual Review of Sociology, 31 (1), 381-399.

Esping-Andersen, G. (1993): Post-industrial class structures: an analytic framework. In: Ders. (Hg.): Changing classes: Stratification and mobility in post-industrial societies. London, Newbury Park, New Delhi: SAGE, 109-135.

Eurofound (2014): Industrial cleaning: Working conditions and job quality. Dublin: Eurofound.

Gather, C./Gerhard, U./Schroth, H./Schürmann, L. (2005): Vergeben und Vergessen? Gebäudereinigung im Spannungsfeld zwischen kommunalen Diensten und Privatisierung. Hamburg: VSA.

Gemeinde Langkampfen (2019): Stellenausschreibung - Reinigungskraft in Teilzeitbeschäftigung. Online: https://www. langkampfen.at/Stellenausschreibung_-_Reinigungskraft_in_Teilzeitbeschaeftigung_7 [07.05.2019].

Geisberger, T. (2017): Niedriglohnbeschäftigung in Österreich 2014: geringe Dynamik - verfestigte Strukturen. Statistische Nachrichten, 11/2017, 924-939.

Herod, A./Aguiar, L.L.M. (2006): Introduction: Cleaners' Agency. In: Aguiar, L.L.M./Herod, A. (Hg.): The Dirty Work of Neoliberalism. Cleaners in the Global Economy. Malden, Oxford, Carlton: Blackwell Publishing, 173-176.

Hofmann, J./Weiss, H. (2018): Klassen messen. „Soziale Klassen" in der empirischen Forschung in Österreich (1945-1990). Österreichische Zeitschrift für Soziologie, 43 (3), 231-250.

Hohnen, P. (2012): Capacities and vulnerabilities in precarious work. The perspective of employees in European low wage jobs. Synthesis report on employees' experience and work trajectories for work package 7 of the walqing project. Roskilde: Roskilde University.

Holtgrewe, U. (2015): Einfachdienstleistungen in der Fläche die Unterseite der Tertiarisierung. Dienstleistungsgestaltung und -politik in schwierigem Gelände. AIS-Studien, $8(1), 5-20$.

Holtgrewe, U./Kirov, V./Ramioul, M. (Hg.) (2015): Hard Work in New Jobs. The Quality of Work and Life in European Growth Sectors. Houndmills: Palgrave Macmillan.

Holtgrewe, U./Sardadvar, K. (2012): Hard work. Job quality and organisation in European low-wage sectors. Synthesis report on company case studies for work package 6 of the walqing project. Vienna: FORBA.

Holtgrewe, U./Sardadvar, K. (2011): Social partnership in unlikely places: The commercial cleaning sector in Austria. walqing social partnership series 2011.1. Vienna: FORBA.

Karafyllis, N.C. (2013): Putzen als Passion. Ein philosophischer Universalreiniger für klare Verhältnisse. Berlin: Kulturverlag Kadmos.

Kirov, V. (2011): How many does it take to tango? Stakeholders' strategies to improve quality of work in Europe. Synthesis report on sector specifics in stakeholder policies and quality of work and life' for work package 5 of the walqing project. Sofia: Institute for the Study of Societies and Knowledge, Bulgarian Academy of Sciences.
Kirov, V. (2015): Can social partners improve the quality of work in low-wage work in Europe? In: Holtgrewe, U./ Kirov, V./Ramioul, M. (Hg.): Hard Work in New Jobs. The Quality of Work and Life in European Growth Sectors. Houndmills: Palgrave Macmillan, 114-129.

Korczynski, M. (2002): Human Resource Management in Service Work. Houndmills: Palgrave.

Krenn, M. (2015): Einfacharbeit - Auslaufmodell oder stabiles Arbeitsmarktsegment? Zur Entmythologisierung der „Wissensgesellschaft" und daraus abgeleiteten Ausgrenzungserfahrungen für gering Qualifizierte. SWS-Rundschau, 55 (2), 150-169.

Krenn, M./Papouschek, U./Gächter, A. (2014): Endbericht zum Forschungsprojekt: Die Verbesserung der Erwerbschancen gering Qualifizierter durch lernförderliche Gestaltung einfacher Arbeit. Wien: FORBA.

Leidner, R. (1996): Rethinking questions of control: Lessons from McDonald's. In: Macdonald, C.L./Sirianni, C. (Hg.): Working in the service society. Philadelphia: Temple University Press, 29-49.

Marchhart, S. (2019): Kollektivvertrag für Angestellte und Lehrlinge in Handelsbetrieben. Wien: Service-GmbH der WKÖ.

März, E. (1968): Die Klassenstruktur der Zweiten österreichischen Republik. In: Leser, N. (Hg.): Probleme österreichischer Politik. Band 1. Wien: Verlag der Wiener Volksbuchhandlung, 7-112.

Mayer-Ahuja, N. (2003): Wieder dienen lernen? Vom westdeutschen „Normalarbeitsverhältnis“ $z$ u prekärer Beschäftigung seit 1973. Berlin: Edition Sigma.

Michenthaler, G./Schaup, T./Bugnar, T. (2013): Beschäftigte im Reinigungsgewerbe. Analyse der Beschäftigteninterviews. Bericht. Wien: IFES.

ÖGB (Österreichischer Gewerkschaftsbund/Gewerkschaft vida) (2018): vida-Katalog für faire Vergabekriterien im Bereich Reinigungsdienstleistungen. Wien: Verlag des ÖGB.

ÖSB (2018): Fair Plus Cleaning. Die Erhebung. Ergebnisse. Wien: ÖSB Consulting GmbH.

Ramioul, M. (2012): Promoting daytime cleaning in Belgium. In: Holtgrewe, U./Sardadvar, K. (Hg.): The Cleaning Sector: Office Cleaning. walqing sector brochures no. 1. Vienna: FORBA.

Ruhland, S./Fürst, C./Gavac, K./Ziniel, W. (2016): Gebäudereinigung in Österreich 2016. Wien. KMU Forschung Austria.

Sardadvar, K. (2012): Indirect paths, fragmented days. Maintenance cleaners in Austria. An internal research report for $W P_{7}$ of the walqing project, SSH-CT-2009-244597. Vienna: FORBA.

Sardadvar, K. (2016): „Schwere Arbeit machen Männer“ Deutungen von Differenz und Strukturen der Ungleichheit in der Reinigungsbranche. Feministische Studien, 2016 (2), 324-340.

Sardadvar, K. (2017): Die Nacht zum Tag gemacht: Das Beispiel Norwegen. Reinigung Aktuell, 16.02.2017. Online: 
http://www.reinigung-aktuell.at/die-nacht-zum-tag-gemacht-das-beispiel-norwegen/ [18.02.2019].

Sardadvar, K./Holtgrewe, U. (2017): Flexibilität mit Grenzen: Büroreinigung im Modus des Gebens und Nehmens. SWS-Rundschau, 57 (2), 211-231.

Sardadvar, K./Kümmerling, A./Peycheva, D. (2015): Gendering work tasks in low-wage sectors. A comparative analysis of commercial cleaning in Austria, domiciliary elderly care in Germany and the waste sector in Bulgaria. In: Holtgrewe, U./Kirov, V./Ramioul, M. (Hg.): Hard Work in New Jobs. The Quality of Work and Life in European Growth Sectors. Houndmills: Palgrave Macmillan, 208-228.

Savage, L. (2006): Justice for Janitors: Scales of Organizing and Representing Workers. In: Aguiar, L.L.M./Herod, A. (Hg.): The Dirty Work of Neoliberalism. Cleaners in the Global Economy. Malden, Oxford, Carlton: Blackwell Publishing, 214-234.

Scandella, F. (2010a): The Cleaners' Revolt: New Trade Union Strategies on Behalf of the Working Poor in England. In: Chabanet, D./Royall, F. (Hg.): Mobilising Against Marginalisation in Europe. Cambridge: Cambridge Scholars Publishing, 195-209.

Scandella, F. (2010b): The hidden face of a booming industry. HesaMag 02, Special report 3/22, Cleaning up: workers under pressure. Brussels: ETUI.

Schroth, H. (2008): Transversale Billigjobber/innen? Dimensionen von Macht und Widerstand im prekären Dienstleistungssektor. In: Marburger Gender-Kolleg (Hg.): Geschlecht, Macht, Arbeit: Interdisziplinäre Perspektiven und politische Intervention. Münster: Westfälisches Dampfboot, 197-210.

Schroth, H./Schürmann, L. (2006): Cleaning Affairs. Geschlechterungleichheiten und Arbeitszeiten im Reinigungsgewerbe. In: Aulenbacher, B./Bereswill, M./Löw, M./Meuser, M./Mordt, G./Schäfer, R./Scholz, S. (Hg.): FrauenMännerGeschlechterforschung. State of the Art. Münster: Westfälisches Dampfboot, 289-299.

Schürmann, L. (2013): Schmutz als Beruf. Prekarisierung, Klasse und Geschlecht in der Reinigungsbranche. Eine wissenssoziologische Untersuchung. Münster: Westfälisches Dampfboot.

Staab, P. (2014a): Macht und Herrschaft in der Servicewelt. Hamburg: Hamburger Edition.

Staab, P. (2014b): „Einfache Dienstleistungen“ in der Wertschöpfungskette. Gegenblende - das DGB-Debattenportal. Online: http://gegenblende.dgb.de/29$2014 /++$ co++141956 d 4-3f $32-11$ e $4-8 f_{9}$ e-52540066f 352 [25.08.2018].

Torvatn, H./Lamvik, G. (2011): A decade in professionalisation in cleaning: MUNICLEAN - a cleaning case study from Norway. Internal report for WP6 of the walqing project, SSH-CT-2009-244597. Trondheim: SINTEF.

Uni Europa/EFCI (2016): Selecting best value. A guide for private and public organisations awarding contracts for cleaning services. Brussels: EFCI/Uni Europa.
vida/WKO (2019): Lohnvereinbarung für Arbeiterinnen/Arbeiter in der Denkmal-, Fassaden- und Gebäudereinigung, im sonstigen Reinigungsgewerbe und in Hausbetreuungstätigkeiten. Stand 1. Jänner 2019. Online: https://www. wko.at/service/kollektivvertrag/lohnvertrag-denkmalfassaden-gebaeudereiniger-2019.pdf [07.05.2019].

Weil, D. (2014): The Fissured Workplace. Why work became so bad for so many and what can be done to improve it. Cambridge, Massachusetts; London, England: Harvard University Press. 